\title{
K ATP Channel-Associated Diabetes Mellitus
}

National Cancer Institute

\section{Source}

National Cancer Institute. KATP Channel-Associated Diabetes Mellitus. NCI Thesaurus.

Code C131847.

Diabetes mellitus caused by activating mutation(s) in genes (KNCJ11 and/or ABCC8) encoding either of the 2 proteins (Kir6.2 and/or SUR1) that make up the pancreatic beta cell adenosine triphosphate-sensitive potassium channel, which is crucial for the regulation of glucose-induced insulin secretion. 